\title{
THE PITUITARY GLAND.*
}

\author{
By S. LEVY SIMPSON, M.A., M.D., M.R.C.P.,
}

(Physician to Willesden General Hospital, Asst. Physician Princess Louise Kensington Hospital for Children; Research Worker Dept. Endocrinology, Lister Institute.)

The pituitary gland has come to be regarded as the master key of the endocrine system in so far as it influences the activity of the remaining glands, but a reciprocal influence in the opposite direction is equally important. Hypophysectomy results in diminished function and some degree of atrophy in the gonads, adrenals, thyroid, pancreas, and less certainly of the parathyroids ; and conversely the chemists have extracted from the anterior pituitary different fractions which stimulate these glands. These factors, e.g. thyrotropic, when injected for some days or weeks produce antibodies in the blood, e.g. antithyrotropic, which inhibit or prevent their activity.

The pituitary is also concerned with growth, adiposity, emaciation, temperature control, diuresis and antidiuresis, sleep, blood pressure, hair and appetite. Extracts can produce diabetes, ketosis, exophthalmos even after thyroidectomy, milk secretion, and melanophore expansion.

The pituitary gland is surrounded by the hypothalamic region of the brain which includes the tuber cinereum, corpora mamillaria, and a large number of separate nerve centre or nuclei. Nearly all the influences of the pituitary gland can be produced by lesions in the neural tissue surrounding the gland and separate centres are being detected for different functions.

Simmonds' cachexia is comparable to hypophysectomy cachexia and is due to destruction of the anterior lobe by idiopathic atrophy, embolus, thrombosis, syphilis, chromophobe adenoma, cranio-pharyngioma, etc. Occasionally it appears to follow influenza, mumps, or chicken pox. The main features are wasting, anorexia, asthenia, sexual atrophy and loss or failure to develop sexual secondary characteristics, hypotension, bradycardia, failure of epiphyseal union in young adults, grey hair, loss of teeth, premature senescence, and progeria.

Cushing's syndrome should be regarded as a clinical entity of varying pathological basis. The main features are loss of sexual function, virilism in the female and feminisation of the male, adiposity, hypertension, polycythæmia, diabetes, lineæ distensæ on the abdomen, acro-cyanosis and bruising of the lower limbs, softening and decalcification of the bones and sometimes exophthalamos. The syndrome is believed by Cushing to be due to a basophil adenoma of the pituitary, but it can also be produced by a tumour of the adrenal cortex or the

* Abstract of Lecture delivered in a course on Endocrinology arranged by Fellowship of Medicine. 
thymus in the absence of a basophilia or a basophil adenoma of the pituitary. A common factor to all conditions is hyalinisation of the basophil cells (Crooke). Since adrenalectomy produces diminution of the basophil cells it is probable that hyperplasia or neoplasm of the adrenal cortex tends to produce basophilia. Thymus tumours are associated with hyperplasia of the adrenal cortex. Radiation of the pituitary gland is of value in Cushing's syndrome.

The increase in the basophil cells of the pituitary also occurs after castration, and at the menapause, and sometimes with hypertension, malignant nephritis, and eclampsia. The latter condition is held by some to be due to an overactivity of the vaso-pressor and anti-diuretic factors and is associated with a relative deficiency of oestrin and excess of prolan. The basophilia that occurs after experimental castration is associated with an increased gonadotropic activity of the pituitary gland, as shown by implantation into another animal. Hence the belief that the basophil cells are the source of pituitary gonadotropic hormone.

Adiposity is still a baffling subject. Experimentally it can be produced by lesions of the tuber cinerum and by injections of chromic acid in the supra-sellar regions. Clinically it may occur with cranio-pharyngiomas (supra-sellar cysts), encephalitis and tabes affecting the hypothalmic region, basophil adenoma, adrenal cortical tumous or hyperplasia, tumours of the third ventrical, secondary hydrocephalus, hyperinsulinism, hypothyroidism, castration of hypogonadism, and at the menapause. But in many cases there is no gross pathological basis. An anterior pituitary extract can produce ketosis; and pitressin produces infiltration of fat into the liver with its subsequent conversion into glycogen. A fat meal produces an increase of blood cholesterol in the obese which is prevented by pitressin. The obese are very prone to starvation creatinuria, suggesting a malfunctioning glycogen metabolism. Water retention may play a part in some forms of apparent adiposity as is illustrated by premenstrual water retention and gain in weight. Although overfeeding may be a factor in some types of adiposity, and although dieting would usually produce some decrease in weight, unmixed so-called exogenous adiposity is probably rare. Pathological hunger comparable to pathological thirst occurs. The B.M.R. is usually normal or sometimes above normal with adiposity. That is why thyroid and dinitrocresol are only of limited value, and the use of the latter is not without danger. A pituitary factor suitable for clinical application is still lacking.

Although development of the breasts is produced by estrin and progestin in a manner comparable to their respective actions on the uterus, actual lactation depends upon the hypophysis, and can not occur in its absence. An extract of anterior lobe (prolactin) produces lactation in castrated male guinea pigs previously treated with ostrin and progestin. Suckling maintains lactation by the nervous stimulus which travels to the pituitary and sets free prolactin. The latter also produces broodiness in fowls and maternal behaviour in rats. Although œestrin and prolactin develop the breasts, both inhibit actual lactation, and a sudden fall in their blood level excites lactation, probably through the release 
of prolactin. Growth is associated with the eosinophile cells and congenital dwarf mice have few eosinophiles. The action of the growth hormone is associated with retention of nitrogen and calcium. Experimental acromegaly can be produced in dogs. The dwarfism of cretins is probably associated with a secondary decrease in the eosinophiles. The great height of castrates results from an overactive pituitary in the presence of ununited epiphyses. In Infantalism both the growth and sex hormones are deficient, and if one produced sexual maturity before giving growth hormone, the epiphyses would unite and further growth be prevented.

The gonadotropic hormone of the pituitary gland consists of two factors $A$ and $B$, both of which have now been chemically separated. A acts on the granulosa cells of the Graaffian follicle producing true maturation, and on the seminiferous tubules of the testis. B acts on the theca cells of the Graaffian follicle, producing luteinisation (incomplete or pseudo in the absence of $\mathrm{A}$, but true after the action of A) and on the interstitial cells of ovary and testis. Pregnancy urine contains chiefly B, but castration and menapausal urine contains A. Hypophysis extracts contain both A and B. (Further essential differences between urinary and hypophysis extracts were referred to.)

Clinically, pregnancy urine extracts (prolan, pregnyl, antuitrin S) are of value in menorrhagias, particularly pre-menapausal, secondary amenorrhœas, threatened abortion, and in bringing undescended testicle into the scrotum.

The injection of pituitrin or pitressin into the third ventricle of the brain produces flushing, vasodilatation, fall of blood pressure, salivation, perspiration, increase of gastric motility, retching, vomiting, fall of basal metabolism and a marked fall of temperature. These effects suggest an action on the cranial autonomic system (Cushing), and they can be minimised by intraventricular injection of pilocarpine and prevented by simultaneous intraventricular injection of atropine. Large doses of pituitrin intramusculary produce gastric erosion and ulcer, and a severe macrocytic anæmia (Dodds).

(Slides illustrating the various conditions were shown and clinical treatment indicated.) 